\title{
NON INVASIVE PRENATAL DIAGNOSIS OF ANEUPLOIDY: NEXT GENERATION SEQUENCING OR FETAL DNA ENRICHMENT?
}

\author{
Webb A, Madgett TE, Miran T, Sillence K, Kaushik N, Kiernan M, Avent ND*
}

*Corresponding Author: Professor Neil D. Avent, School of Biomedical and Biological Sciences, Faculty of Science and Technology, A411 Portland Square, Drake Circus, Plymouth, Devon, PL4 8AA, UK; Tel.: +44(0)1752-584884; Fax: +44-(0)1752-584605; E-mail: neil.avent@plymouth.ac.uk

\begin{abstract}
Current invasive procedures [amniocentesis and chorionic villus sampling (CVS)] pose a risk to mother and fetus and such diagnostic procedures are available only to high risk pregnancies limiting aneuploidy detection rate. This review seeks to highlight the necessity of investing in non invasive prenatal diagnosis (NIPD) and how NIPD would improve patient safety and detection rate as well as allowing detection earlier in pregnancy.

Non invasive prenatal diagnosis can take either a proteomics approach or nucleic acid-based approach; this review focuses on the latter. Since the discovery of cell free fetal DNA (cffDNA) and fetal RNA in maternal plasma, procedures have been developed for detection for monogenic traits and for some have become well established (e.g., RHD blood group status). However, NIPD of aneuploidies remains technically challenging. This review examines currently published literature evaluating techniques and approaches that have been suggested and developed for aneuploidy detection, highlighting their advantages and limitations and areas for further research.
\end{abstract}

Keywords: Aneuploidy; Cell free fetal DNA (cff DNA); Non invasive prenatal diagnosis (NIPD).

School of Biomedical and Biological Sciences, University of Plymouth, Plymouth, Devon, UK

\section{INTRODUCTION}

Current invasive diagnostic techniques pose a risk to mother and fetus. The National Health Service (NHS) offers prenatal screening to all pregnant women in England [1]. At 11 to 13 weeks gestation a combination of tests (termed the combined test) are performed to screen for abnormalities and score the risk of the fetus having Down's syndrome (trisomy 21) (T21). The combined test includes ultrasound scans to check nuchal translucency and analyzing maternal blood samples for free $\beta$ human chorionic gonadotrophin and pregnancy-associated plasma protein A concentrations. These are combined with factors such as maternal age, pregnancy histories and familial genetic conditions [1]. Women with high risk pregnancies are then offered prenatal diagnosis. Current diagnostic procedures take samples for karyotyping through invasive means, posing a risk to mother and fetus.

Chorionic villus sampling (CVS) can be performed from 10 weeks; taking a tissue sample from the placenta through the cervix or abdomen. Amniocentesis can be performed from 15 weeks by obtaining a sample of amniotic fluid by passing a needle through the mother's abdomen into the uterus [1]. The rate of miscarriage associated with CVS and amniocentesis is 1.0 to $2.0 \%$. Despite the risks, on average $5.0-10.0 \%$ of pregnant women chose to undergo these tests [2], averaging approximately 
23,700 invasive diagnostic tests per year [1]. The combined test has a $5.0-9.0 \%$ false positive rate $[3,4]$; consequently mothers with healthy fetuses may choose to undergo unnecessary invasive diagnostic procedures, putting the fetus at risk of iatrogenic spontaneous abortion. Non invasive prenatal diagnosis (NIPD) would pose no such risk.

Increasing maternal age increases risk of Down's syndrome, Edwards' syndrome (trisomy 18) (T18) and Patau's syndrome (trisomy 13) (T13), the three most common autosomal aneuploidies in live births. In the early $1970 \mathrm{~s}$, about $5.0 \%$ of pregnant women were 35 years or over. However, almost $20.0 \%$ are now over 35 , increasing the incidence of aneuploid fetuses [5]. Trisomy 18 and T13 are currently not screened for until fetal anomaly ultrasound screening at 18 to $20^{+6}$ weeks [1]. However, the NHS state that although abortion is legal up to 24 weeks, it should be carried out as early as possible, ideally before 12 weeks [6]. Therefore, preferably, detection of all aneuploidies would be made within the first trimester, which would give greater scope for parental choice. It is hoped that NIPD could help achieve this.

Despite increasing maternal age, approximately $80.0 \%$ of Down's syndrome patients are stillborn to those under 35 [7]. Currently diagnostic tests are only offered to those with high risk pregnancies (such as those with abnormal serum protein levels) as the risk of having a fetus with an abnormality must be balanced against the risk of miscarriage. This inevitably results in some abnormalities being missed as women under 35 years are not considered at risk of having a Down's syndrome fetus [7]. Non invasive prenatal diagnosis would allow testing of these low-risk pregnancies. A recent European Commission (EC) funded project, SAFE (Special non-invasive advances in fetal and neonatal evaluation) explored a number of new technologies in NIPD [8,9], and this has been extended in a recent project funded by the UK National Institute for Health Research (NIHR), RAPID, and a EC framework 7 program, Eurogentest 2.

In summary, prenatal testing is important as it allows possible health issues of the baby to be identified before birth, allowing arrangements for immediate care to be made. It also enables parents to make an informed choice regarding whether to terminate the pregnancy. Replacing current invasive tests with NIPD would reduce risk and increase detection rate.

Methods for Non Invasive Prenatal Diagnosis of Aneuploidies. The importance of prenatal diagnosis and risk posed by invasive techniques makes NIPD research a worthwhile commitment morally and commercially. Research originally focused on fetal nucleated cells found in maternal circulation [10-12]. A number of cell types were investigated but subsequently ruled out for a variety of reasons. These include lack of fetal specific markers [13], entrapment in the maternal lungs, confined placental mosaicism [14] and persistence in the maternal circulation years after pregnancy [15]. Furthermore, only one or two fetal nucleated cells are found per $\mathrm{mL}$ of maternal blood, further hampering their use [7]. Research now focuses on cell free fetal DNA (cffDNA), which was discovered in maternal plasma in 1997 [10]. It has since been reported to be present from as early as 4 weeks gestation [16], making it possible for NIPD to be achievable earlier in pregnancy than invasive methods. It has a short circulation half-life ( $\approx 16 \mathrm{~min}$.) and is undetectable in the maternal circulation within 2 hours postpartum [17], making it specific to the current pregnancy. Technical challenges stem from the fact cffDNA constitutes only 3.0 to $6.0 \%$ of cell free DNA in maternal plasma [7,10]. Detection of paternally inherited alleles in maternal plasma is qualitative; however, direct detection of aneuploidies is reliant on dose, therefore quantitative. Therefore, it was originally assumed that direct measurement of fetal chromosome dose in maternal plasma would show maternal chromosome dose and fetal chromosome dose would be lost in the background of maternal DNA. Nevertheless, chromosome dose methods have been developed and are discussed later. Methods for aneuploidy detection originally focused on fetal markers for allelic ratio analysis.

Non Invasive Prenatal Diagnosis in Routine Clinical Practice 2012. Following the discovery of cffDNA in maternal plasma in 1997 [10], testing for fetal RHD blood group status rapidly evolved from risky DNA testing of amniotic fluid samples provided from Liley curve investigations [18] to the world's first routine application of a non invasive prenatal test clinically [19]. Fetal blood grouping using maternal plasma as a source of fetal DNA is now used extensively worldwide [20]. In addition, 
the detection of other paternally inherited alleles or chromosome is possible, for example, fetal sexing (for review, see [21]). Most clinically applicable methods utilize the simple real time polymerase chain reaction (ReTi-PCR) approach, however, methods to detect the most commonly requested prenatal diagnostic test, for aneuploidy, require more sophisticated approaches. This is primarily due to the fact that maternal DNA is an admix of fetal (derived from the placenta) and maternal DNA.

\section{METHODS TO DETECT FETAL ANEUPLOIDY BY NON INVASIVE PRENATAL DIAGNOSIS}

Methods Based on Allelic Ratio: RNA-Single Nucleotide Polymorphism Allelic Ratio Approach. The presence of free fetal RNA (ffRNA) in maternal plasma was established in 2000 by Poon et al. [22] who showed that $\mathrm{Y}$ chromosome-specific zinc finger protein $(Z F Y)$ mRNA could be found in the plasma of women carrying a male fetus. Further studies showed that ffRNA is surprisingly stable [23] and present in maternal plasma as early as 4 weeks gestation [24]. As different tissues express their own individual mRNA profiles, it follows that some mRNAs may be placenta-specific and therefore fe- tal specific. $\mathrm{Ng}$ et al. [25] showed that placenta specific mRNAs could be detected in maternal plasma, and in 2007, a placental-specific mRNA transcribed from PLAC4 on chromosome 21 was discovered [26]; use of these could circumvent the problems caused by maternal background DNA.

Lo et al. [26] developed the RNA-SNP (single nucleotide polymorphism) allelic ratio approach. This technique exploits SNPs that cause sequence variation between alleles. A heterozygous euploid fetus should yield equal proportions of each allele, giving an allelic ratio of 1:1. However, a heterozygous triploid fetus would yield the allelic ratio $1: 2$ or 2:1 [27]. Lo et al. [26] used reverse transcription (RT)-PCR to amplify a PLAC4 SNP containing locus, followed by primer extension. Mass spectrometry was then used to determine allelic ratio (Figure 1). Maternal plasma of 57 women carrying euploid fetuses and 10 carrying T21 fetuses was analyzed. The method had a sensitivity of $90.0 \%$ and specificity of $96.0 \%$ [26]. Larger trials to refine the normal reference ranges could potentially improve sensitivity and specificity [27]. Regrettably, RNA-SNP allelic ratio methods are limited to fetuses heterozygous for the SNP under analysis. Among 119 placentas genotyped by Lo et al. [26] for the most common PLAC4 SNP (rs8130833), 42.0\% were homozygous and the method would have therefore been uninformative.

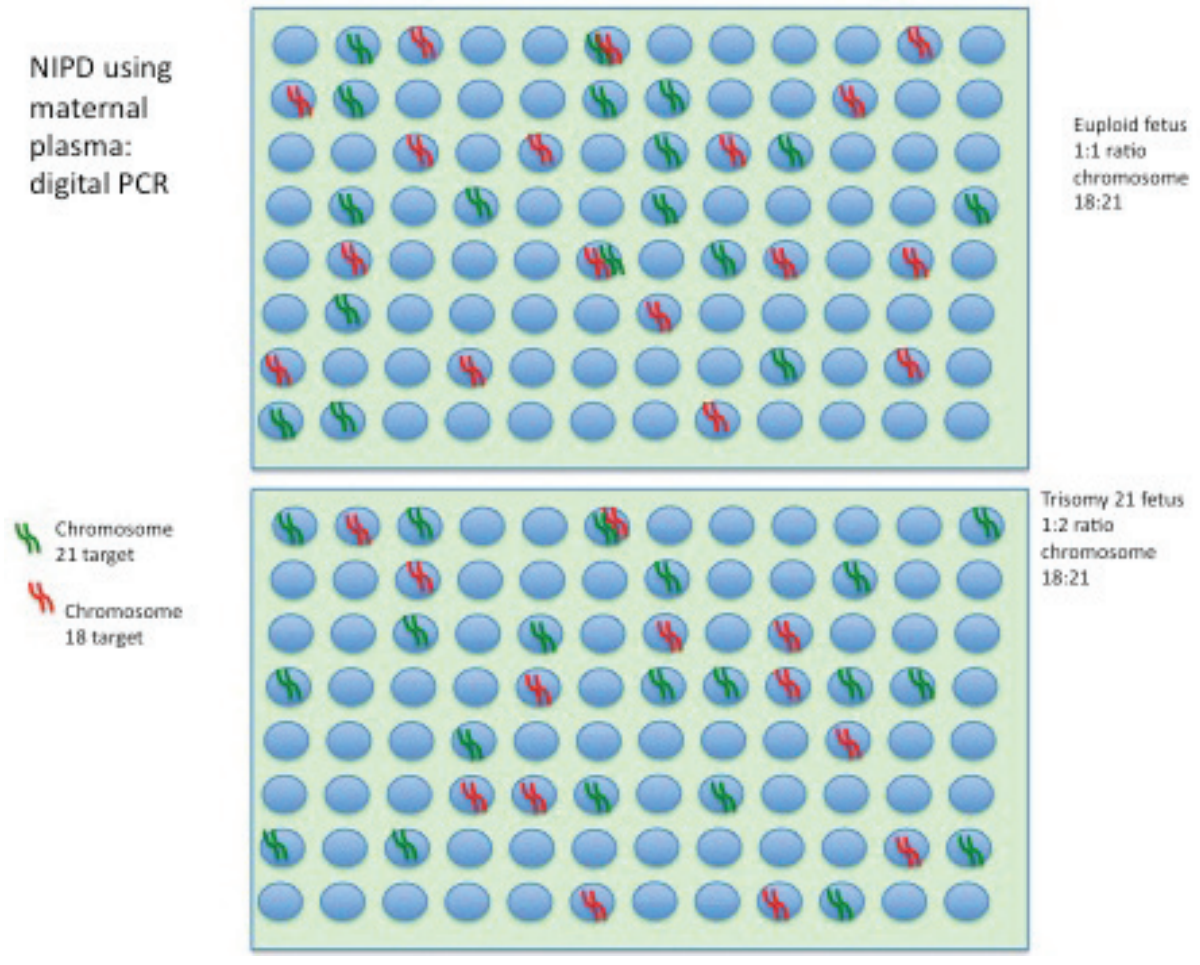

Figure 1. Schematic representation of Digital PCR. 
The Digital RNA-Single Nucleotide Polymorphism Allelic Ratio Approach. The digital RNA-SNP allelic ratio approach is an adaptation to the RNA-SNP allelic ratio method made to utilize the sensitivity of digital PCR [28]. Rather than one reaction mix, digital PCR involves thousands of reactions running in parallel. The template strand solution is diluted so that a maximum of one template strand is added to each reaction. Lo et al. [28] used 384-well plates for digital ReTi-PCR of the PLAC4 SNP, rs8130833. Uninformative wells (i.e., those containing no or multiple signals) were discounted and wells containing A or G PLAC4 allele were counted and their ratio calculated. Using maternal plasma RNA samples, four T21 fetuses and nine euploid were correctly identified. However, although digital PCR is more sensitive than ReTi-PCR, the digital RNA-SNP allelic ratio approach it is still limited to heterozygous fetuses. The methodology entered commercial trials under the governance of Sequenome Inc. (San Francisco, CA, USA) but was subsequently found to be an unreliable technology and unlikely to see routine application in NIPD.

Epigenetic Allelic Ratio Approach. The epigenetic allelic ratio (EAR) approach is similar to the RNA-SNP allelic ratio approach but rather than targeting fetal specific mRNA, it exploits epigenetic phenomena that alter DNA expression without altering sequence; the most well known of these is cytosine methylation. Methylation patterns differ between tissues; genes that are differentially methylated between mother and fetus have been identified allowing an opportunity to selectively target fetal-specific DNA with the use of methylation specific primers [22,27].

Chim et al. [29] demonstrated that the maspin gene (SERPINB5) promoter is unmethylated (U-mapsin) in the placenta but hypermethylated in maternal blood cells. SERPINB5 is located at $18 \mathrm{q} 21.33$ [30], providing the opportunity for an EAR-based approach to T18 detection. Tong et al. [30] used the EAR approach, first using bisulphite conversion followed by methylation-specific PCR and primer extension reactions designed to distinguish the $\mathrm{A}$ and $\mathrm{C}$ allele of $\mathrm{U}$-mapsin based on size [30]. The test had a sensitivity of $100.0 \%$ but had a $9.7 \%$ false positive rate [30]. Theoretical modelling suggested that 200 sequence copies were needed at the start of PCR to achieve diagnostic sensitivity and specificity of $97.0 \%$. However, bisulphite conversion has been reported to cause DNA degradation of up to $96.0 \%$ [30]. Taking this into account, Tong et al. [30] predicted that only 20 sequence copies would have remained in their samples after bisulphite conversion due to low blood volume used; this could explain the high false positive rate.

Although the EAR approach was successfully demonstrated, strategies need to be developed to overcome DNA degradation by bisulphite conversion. Weber et al. [31] described a way of enriching methylated DNA using immunoprecipitation with the use of an antibody against 5-methylcytosine; methylated DNA immunoprecipitation (MeDiP) has since been used to successfully enrich fetal hypermethylated DNA [32]. Alternatively, degradation of maternal unmethylated DNA by bisulphite conversion would be acceptable so targeting genes hypermethylated in the placenta but hypomethylated in maternal tissues could be a solution [3].

Despite various groups showing methods based on allelic ratio to work successfully, all are limited to heterozygous fetuses. However, an allelic ratio method could still have potential for use in practice if multiple SNPs, with a combined heterozygosity rate high enough to cover the general population, were analyzed [27]. Allelic ratio methods also assume both alleles are transcribed at an equal rate, which is not always true [4], meaning analysis of transcript ratios could be unreliable. However, successful demonstration of allelic ratio methods suggests this is not problematic. Finally, although allelic ratios may successfully identify trisomies they would not detect monosomies.

Methods Based on Relative Chromosome Dosage. Using microfluidics digital PCR, Lun et al. [33] re-evaluated the percentage of cell free DNA that was fetal in maternal plasma and found there was a median $9.7 \%$ in the first trimester rising to $20.4 \%$ by the third trimester. These levels are higher than previously thought, suggesting direct measurement of fetal chromosome dose may be possible without prior enrichment. Relative chromosome dose approaches work on the principal that in a normal fetus the ratio of two chromosomes should be 2:2. Comparing an affected chromosome in a triploid fetus would give the ratio 3:2. A number of methods based on chromosome dose have been reported and are described below. 
Epigenetic-Genetic and Epigenetic-Epigenetic Chromosome Dosage Approaches. Tong et al. [34] combined a methylation restricted digest to digest hypomethylated DNA and microfluidics digital PCR to measure $H L C S$ on chromosome 21 (a hypermethylated fetal marker), RASSF1A on chromosome 3 (a hypermethylated fetal marker) and $Z F Y$ on the $Y$ chromosome. The epigenetic-epigenetic chromosome dosage approach comparing hypermethylated HLCS and RASSF $1 A$ ratio showed too great an overlap between euploid and T21 fetuses. However the epigenetic-genetic chromosome dosage approach comparing hypermethylated $H L C S$ and $Z F Y$ ratio in euploid and T21 fetuses was discriminative but limited to male fetuses. Other groups have also successfully used the EGG chromosome dose approach, for example, Tsui et al. [35] identified T18 fetuses with a sensitivity of $88.9 \%$ and specificity of $96.3 \%$. However, Tsui et al. [35] also used ZFY limiting use to male fetuses. $Z F Y$ is often used for proof of principal studies but epigenetic-genetic (EGG) chromosome dosage needs to be successfully demonstrated with sex-independent markers before it can be used in practice.

Digital Relative Chromosome Dosage Method. There is a 1.5 -fold increase in relative chromosome dose in trisomies. Due to the exponential increase in template strand number in ReTi-PCR, it is not suited to detecting differences in template strand concentrations below 2-fold [4,36,37]. Digital PCR provides a more accurate measure of template strand concentration.

Methodology involves the amplification of multiple targets on different chromosomes in many thousands of different PCR reactions (microfluidic PCR or droplet PCR). In this example, two regions of the human genome have been amplified, chromosomes 18 and 21. The relative abundance of the target DNA is defined directly against one another, and thus a statistical 1:1 ratio of each chromosome would indicate a euploid fetus and a (theoretical) 2:1 ratio (21:18) would indicate aneuploidy. Despite the admix of fetal and maternal DNA in maternal plasma, like next generation sequencing (NGS) (see Figure 2), digital PCR is able to differentiate such a difference, although clearly the actual difference in ratio would not be $2: 1$ due to the presence of maternal DNA.

The digital relative chromosome dosage (RCD) method directly targets a non polymorphic locus on

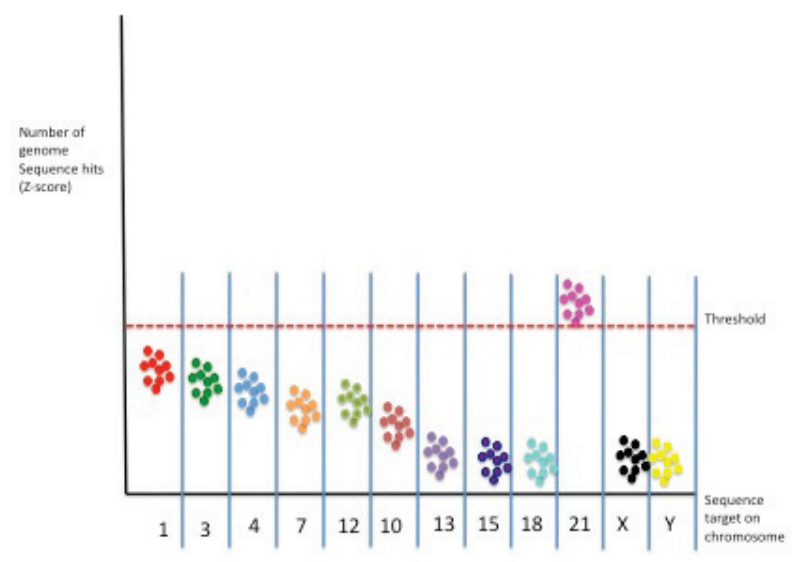

Figure 2. Schematic representation of next-generation sequencing based approaches for NIPD of fetal aneuploidy. The figure represents the numbers of targets from each chromosome (X-axis) identified by NGS as colored dots. Based on statistical counting of each chromosome (Z-score), a higher proportion of chromosome 21 signals can be shown in maternal plasma samples despite this being an admix of maternal and fetal DNA. The approach can include whole genome sequencing, or targeted sequencing of various chromosomes following amplification the chromosome of interest and on a reference chromosome without differentiating between maternal and fetal DNA. Lo et al. [28] used digital PCR to measure a non polymorphic locus on chromosome 21 and chromosome 1. The number of wells in which there was a positive PCR for either locus was counted and their relative dose calculated. Euploid and T21 fetuses in artificial mixtures of placental and maternal DNA were successfully identified but the method was not tested using maternal plasma from T21 pregnancies. However, despite RCD not differentiating between maternal and fetal DNA, computer modeling estimated $97.0 \%$ of fetuses would be correctly identified by 7680 digital PCRs in cases where the cffDNA constituted $25.0 \%$ of the DNA [28]. Nevertheless, this is higher than previously reported levels in maternal plasma. Moreover, other groups have shown the digital RCD approach to be feasible with fetal fractions of $10.0 \%$ [38] although some level of enrichment may still first be needed for use in the first trimester.

Next Generation Sequencing (NGS) or Massively Parallel Sequencing. Massively parallel sequencing (MPS) is a method through which the entire genome can be sequenced using millions of short sequence reads. These are then reassembled by a computer program using genomic databases to compare the reads to the known genomic sequence $[3,4,39]$. A number of groups have demonstrated 
that if cffDNA is sequenced in this way and the sequence reads assigned to each chromosome are then counted, whether a chromosome is over- or underrepresented can then be calculated $[39,40]$. This is similar to the approach taken for the digital relative chromosome dose method. However, NGS generates far more sequence reads, possibly over 60,000 for chromosome 21 alone, suggesting it should be more sensitive to small changes in genomic representation [4]. The NGS could therefore have the potential of identifying partial trisomies, although further research is needed to investigate this.

Fan et al. [39] were able to identify nine T21 cases, two T18 and one T13, distinguishing them from six normal euploid cases, using shot gun sequencing (Figure 2). However, the blood samples used in this study were drawn 15 to $30 \mathrm{~min}$. following an invasive procedure, this may have influenced the results by introducing additional fetal DNA into the maternal circulation [3]. Furthermore, Fan et al. [39] argue that using a digital PCR assay, they estimated fetal DNA in their samples constituted $<10.0 \%$ of total cell free DNA, in line with previous studies.

More recent studies have taken into account that differing GC contents between chromosomes results in nonuniform sequencing by MPS and corrected genomic representation to account for $\mathrm{CG}$ content. By doing this, Chen et al. [40] found that a specificity and sensitivity of 98.9 and $100.0 \%$, respectively, could be achieved for T13 detection and 98.0 and $91.9 \%$, respectively for T18 detection.

In light of recently successful studies, Ehrich et al. [41] conducted a blinded study on 449 plasma samples from pregnant women (39 with T21 fetuses), using the most up-to-date sequencing technology available at the time, to investigate NGS for T21 diagnosis. Z-scores described in [42] were used to standardize genomic representation and classify the fetus as euploid or T21. The results were very successful with a sensitivity of $100.0 \%$ and specificity of $99.7 \%$, suggesting that if the cost of MPS could be reduced it could be used in practice. Recently, using the single molecule sequencing technology of Helicos (Cambridge, MA, USA) that does not require a prior DNA amplification step, van den Oever et al. [43] have demonstrated a greater sensitivity of this platform above that of Illumina GAII (San Diego, CA, USA) used in previous studies. This may lead to utilization of NGS much earlier in
NIPD, perhaps within the first trimester [44], as the study demonstrated detection of trisomy 21 .

All three relative chromosome dose methods discussed are polymorphism-independent and have successfully demonstrated the ability to detect trisomies. Of the three relative chromosome dose methods, NGS seems the most promising as, due to the large amount of data it produces, it has high sensitivity and specificity. It can also simultaneously provide information on chromosome dose for all chromosomes, and theoretically, has the potential to detect partial trisomies and monosomies, although this needs to be validated by further research.

Methods to Enrich Fetal DNA. One of the main hindrances on NIPD is the dilution of fetal DNA in maternal blood; this makes the quantitative nature of aneuploidy diagnosis difficult. Therefore, a number of methods have been investigated to enrich or prevent the dilution of fetal DNA in maternal plasma.

Use of Formaldehyde. In 2004, Dhallan et al. [45] hypothesized that a significant portion of maternal DNA in maternal plasma is leaked from maternal leukocytes following venipuncture due to physical forces put on them during collection and subsequent handling. Blood was collected from 69 pregnant women in tubes containing a $4.0 \%$ formaldehyde neutralizing buffer. Analysis showed the majority of samples had $>25.0 \%$ fetal DNA and $27.5 \%$ had $>50.0 \%$, suggesting formaldehyde treatment could successfully prevented leukocyte rupture. However, no untreated blood was collected in this trial so no comparisons between treated and untreated samples could be made. Since 2004, a number of groups have attempted to replicate the enrichment of fetal DNA in maternal plasma using formaldehyde without success [46,47].

Separation Based on Size by Gel Electrophoresis. Another approach to fetal enrichment is to separate cffDNA from maternal based on size. This can be done by gel electrophoresis [48]. In early pregnancy $(13+2$ weeks gestational ages), Li et al. [48] found that $85.5 \%$ of fetal DNA is less than $0.3 \mathrm{~kb}$. Fetal DNA also constituted $28.4 \%$ of the $<0.3 \mathrm{~kb}$ fraction in maternal plasma, increasing to $68.7 \%$ in the third trimester. A study by Chan et al. [49] supports Li et al. [48], concluding that $>99.0 \%$ of fetal derived DNA is shorter than $313 \mathrm{bp}$. Gel electrophoresis has since been used to successfully enrich fetal DNA for detection of point mutation in 
$\beta$-thalassemia [50]. However, isolation of size fractions by gel electrophoresis is considered too time consuming and prone to contamination to allow its widespread use [3].

Co-Amplification at Lower Denaturation Temperature Polymerase Chain Reaction. In 2008, Li et al. [51] reported on (co-amplification at lower denaturation temperature) COLD-PCR, a variation of PCR that can selectively amplify minority alleles from a background of wild-type alleles. This technique works on the basis that even a single nucleotide difference between the minority and wild-type allele may lower the critical denaturation temperature $\left(T_{c}\right)$. If so, the minority allele could be denatured at a lower temperature than the wild-type sequence, allowing only the minority allele sequence to bind with the primers and be amplified [52]. Li et al. [51] used COLD-PCR to identify mutations in a number of genes associated with human cancer that had previously been missed and suggested that COLD-PCR could also be used for detection of "fetal alleles in maternal blood." However, in aneuploidies there may be no sequence difference between the maternal and fetal allele of interest. Moreover, it is possible that the shorter length of cffDNA in comparison with maternal cell free DNA may allow COLD-PCR to denature cffDNA at a temperature at which maternal DNA would remain double-stranded, allowing only cffDNA to be amplified. There is currently no published study on the enrichment of fetal DNA from maternal plasma using this method but this could be a promising technique. If fetal DNA could be enriched in this way, not only could it aid detection of aneuploidies but also aid detection of monogenic diseases where a disease allele may have been maternally inherited.

Proteomics for New Down's Syndrome Biomarkers (for review, see [53,54]). Despite the advances in molecular counting technologies, especially NGS and digital PCR, better serum screening biomarkers for Down's syndrome and other conditions are still an option, as NGS and digital PCR methodologies are not yet applicable to mass-scale screening. For this reason several large scale studies were launched to analyze the maternal plasma proteome in pregnancies where the mother was carrying a Down's syndrome fetus [53,55-60]. It is clear that a number of overlapping biomarkers have been identified, but the search for a truly differentiating trisomy
21 biomarker using this approach may still be a way off, but may well be worth the effort expended.

\section{CONCLUSIONS}

In summary, despite various non invasive methods invented to diagnose aneuploidy, none are yet used in practice. Methods described in the current literature can be split into methods based on allelic ratio or chromosome dose. Allelic ratio methods are held back by their restriction to heterozygous fetuses, yet if a combination of SNPs could be used, their combined heterozygosity rate could potentially cover the general population. However, allelic ratio approaches would still be unable to diagnose monosomies. Chromosome dose methods have the advantage of being polymorphismindependent and have the potential to diagnose trisomies, monosomies and in the case of NGSbased methods, possibly even partial trisomies but this needs to be verified by further research. For these methods to be introduced into practice, further research needs to be aimed at simplifying the methods and reducing the cost. Another approach would be to selectively enrich cffDNA or deplete maternally derived cell free DNA to overcome the problem of low cffDNA concentration in maternal plasma; this would allow more straight-forward analysis methods to be used. Co-amplification at lower denaturation temperature-PCR seems the most promising enrichment technique discussed but requires further investigation.

With the progress made in NIPD since the discovery of cffDNA, and invention of new sequencing techniques, it is not over optimistic to predict that NIPD will be used in practice in the near future; improving detection rate, allowing earlier diagnosis and eliminating iatrogenic fetal loss and risk to the mother due to invasive procedures.

\section{REFERENCES}

1. NHS FASP, 2010. NHS Fetal Anomaly Screening Programme Annual Report 2009-2010. Available at $\quad[\mathrm{http}: / /$ fetalanomaly.screening.nhs.uk/getdata. php?id=11354] Accessed on January 162012.

2. Mujezinovic F, Alfirevic Z. Procedure-related complications of amniocentesis and chorionic villous sampling. Obstet Gynecol. 2007; 110(3): 687-694.

3. Go AT, van Vugt JMG, Oudejans CBM. Non- 
invasive aneuploidy detection using free fetal DNA and RNA in maternal plasma: recent progress and future possibilities. Hum Reprod Update. 2011; 17(3): 372-382.

4. Hahn S, Lapaire O, Tercanli S, Kolla V, Hösli I. 2011. Determination of fetal chromosome aberrations from fetal DNA in maternal blood: has the challenge finally been met? Expert Rev Molec Med. 2011; 13; 16 : e16. Available at [http://www.ncbi.nlm.nih.gov/pmc/ articles/ PMC3087311/pdf/S1462399411001852a. pdf] Accessed on January 162012.

5. Nicolaides KH. Screening for fetal aneuploidies at 11 to 13 weeks. Prenat Diagn. 2011; 31(1): 7-15.

6. NHS, 2010. When an abortion should be carried out. Available at [http://www.nhs.uk/Conditions/ Abortion/ Pages/When-should-it-be-done.aspx] Accessed on January 82012.

7. Bischoff FZ, Sinacori MK, Dang DD, et al. Cell-free fetal DNA and intact fetal cells in maternal blood circulation: implications for first and second trimester non-invasive prenatal diagnosis. Hum Reprod Update. 2001; 8(6): 493-500.

8. Chitty LS, van der Schoot CE, Hahn S, Avent ND. SAFE-The special non-invasive advances in fetal and neonatal evaluation network: aims and achievements. Prenat Diagn. 2009; 28(2): 83-88.

9. Maddocks DG, Alberry MS, Attilakos G, et al. The SAFE project-towards non-invasive prenatal diagnosis. Biochem Soc Transactions. 2009; 37: 460-465.

10. Lo YMD, Corbetta N, Chamberlain PF, et al. Presence of fetal DNA in maternal plasma and serum. Lancet 1997; 350(9076): 485-487.

11. Lo YMD. Non-invasive prenatal diagnosis using fetal cells in maternal blood. J Clin Pathol. 1994; 47(12): 1060-1065.

12. Herzenburg LA, Bianchi DW, Schroder J, Cann HM, Iverson GM. Fetal cells in the blood of pregnant women: Detection and enrichment by fluorescenceactivated cell sorting. Proc Natl Acad Sci USA. 1979; 76(3): 1453-1455.

13. Avent ND, Plummer ZE, Madgett TE, et al. Postgenomics studies and their application to non-invasive prenatal diagnosis. Semin Fetal Neonat Med. 2008; 13: 91-98.

14. Wapner RJ. Invasive prenatal diagnostic techniques. Semin Perinatol. 2005; 29(6): 401-404.

15. Bianchi DW, Zickwolf GK, Weil GJ, Sylvester S, DeMaria MA. Male fetal progenitor cells persist in maternal blood for as long as 27 years postpartum. Proc Natl Acad Sci USA. 1996; 93(2): 705-708.

16. Illanes S, Denbow M, Kailasam C, Finning K, Soothill PW. Early detection of cell-free fetal DNA in maternal plasma. Early Hum Dev. 2007; 83(9): 563-566.
17. Lo YMD, Zhang J, Leung TN, Lau TK, Chang AM, Hjelm NM. Rapid clearance of fetal DNA from maternal plasma. Am J Hum Genet. 1999; 64(1): 218-224.

18. Avent ND, Finning KM, Martin PG, Soothill PW. Prenatal determination of fetal blood group status. Vox Sang. 2000; 78(2): 155-162.

19. Finning KM, Martin PG, Soothill PW, Avent ND. Prediction of fetal D status from maternal plasma: introduction of a new noninvasive fetal RHD genotyping service. Transfusion. 2002; 42(8): 1079-1085.

20. Scheffer PG, van der Schoot CE, Page-Christiaens GCML, de Haas M. Noninvasive fetal blood group genotyping of rhesus D, c, E and of $\mathrm{K}$ in alloimmunised pregnant women: evaluation of a 7 year clinical experience; Br J Obstet Gynaecol. 2011; 118(11): 1340-1348.

21. Avent ND, Chitty LS. Non-invasive diagnosis of fetal sex; utilisation of free fetal DNA in maternal plasma and ultrasound. Prenat Diagn. 2006; 26(7): 598-603.

22. Poon LLM, Leung TN, Lau TK, Chow KCK, Lo YMD. Differential DNA methylation between fetus and mother as a strategy for detecting fetal DNA in maternal plasma. Clin Chem. 2002; 48(1): 35-41.

23. Tsui NBY, Ng EKO, Lo YMD. Stability of endogenous and added RNA in blood specimens, serum, and plasma. Clin Chem. 2002; 48(10): 1647-1653.

24. Chiu RW, Lui WB, Cheung MC. et al. Time profile of appearance and disappearance of circulating placenta-derived mRNA in maternal plasma. Clin Chem. 2006; 52(2): 313-316.

25. Ng EKO, Tsui NBY, Lau TK, et al. mRNA of placental origin is readily detectable in maternal plasma. Proc Natl Acad Sci USA. 2003; 100(8): 4748-4753.

26. Lo YMD, Tsui NBY, Chiu RWK, et al. Plasma placental RNA allelic ratio permits noninvasive prenatal chromosomal aneuploidy detection. Nat Med. 2007; 13(2): 218-223.

27. Lo YMD. Noninvasive prenatal detection of fetal chromosomal aneuploidies by maternal plasma nucleic acid analysis: a review of the current state of the art. Br J Obstet Gynaecol. 2008; 116(2): 152-157.

28. Lo YMD, Lun FMF, Chan KCA, et al. Digital PCR for the molecular detection of fetal chromosomal aneuploidy. Proc Natl Acad Sci USA. 2007; 104(32): 13116-13121.

29. Chim SSC, Tong YK, Chiu RWK, et al. Detection of the placental epigenetic signature of the maspin gene in maternal plasma. Proc Natl Acad Sci USA. 2005; 102(41): 14753-14758.

30. Tong YK, Ding C, Chiu RWK, et al. Noninvasive prenatal detection of fetal trisomy 18 by epigenetic allelic ratio analysis in maternal plasma: theoretical and empirical considerations. Clin Chem. 2006; 52(12): 2194-2202. 
31. Weber M, Davies JJ, Wittig D, et al. Chromosomewide and promoter-specific analyses identify sites of differential DNA methylation in normal and transformed human cells. Nat Genet. 2005; 37(8): 853-62.

32. Papageorgiou EA, Fiegler H, Rakyan V, et al. Sites of Differential DNA Methylation between Placenta and Peripheral Blood: Molecular Markers for Noninvasive Prenatal Diagnosis of Aneuploidies. Am J Pathol. 2009; 174(5): 1609-1618.

33. Lun FMF, Chiu RWK, Chan KCA, et al. Microfluidics digital PCR reveals a higher than expected fraction of fetal DNA in maternal plasma. Clin Chem. 2008; 54(10): 1664-1672.

34. Tong YK, Jin S, Chiu RWK, et al. Noninvasive prenatal detection of trisomy 21 by an epigenetic-genetic chromosome-dosage approach. Clin Chem. 2010; 56(1): 90-98.

35. Tsui DWY, Lam YMD, Lee WS, et al. Systematic identification of placental epigenetic signatures for the noninvasive prenatal detection of Edward's syndrome. PLoS One 2010; 5(11): e15069.

36. Vogelstein B, Kinzler KW. 1999. Digital PCR. Proc Natl Acad Sci USA. 1999; 96(16): 9236-9241.

37. Zimmermann BG, Grill S, Holzgreve W, et al. Digital PCR: a powerful new tool for noninvasive prenatal diagnosis? Prenat Diagn. 2008; 28(12): 1087-1093.

38. Fan HC, Quake SR. Detection of aneuploidy with digital polymerase chain reaction. Anal Chem. 2007; 79(19): 7576-7579.

39. Fan HC, Blumenfeld YJ, Chitkara U, et al. Noninvasive diagnosis of fetal aneuploidy by shotgun sequencing DNA from maternal blood. Proc Natl Acad Sci USA. 2008; 105(42): 16266-16271.

40. Chen EZ, Chiu RWK, Sun H, et al. Noninvasive prenatal diagnosis of fetal trisomy 18 and trisomy 13 by maternal plasma DNA sequencing. PLoS One 2011; 6(7): e21791.

41. Ehrich M, Deciu C, Zwiefelhofer T, et al. Noninvasive detection of fetal trisomy 21 by sequencing of DNA in maternal blood: a study in a clinical setting. Am J Obstet Gynecol. 2011; 204(3): 205.e1-11.

42. Chiu RWK, Chan KCA, Gao Y, et al. Noninvasive prenatal diagnosis of fetal chromosomal aneuploidy by massively parallel genomic sequencing of DNA in maternal plasma. Proc Natl Acad Sci USA. 2008; 105(51): 20458-20463.

43. van den Oever JM, Balkassmi S, Verweij EJ, et al. Single molecule sequencing of free DNA from maternal plasma for noninvasive trisomy 21 detection. Clin Chem. 2012; 58(4): 699-706.

44. Avent ND. Refining noninvasive prenatal diagnosis with single-molecule next generation sequencing. Clin Chem. 2012; 58(4): 657-658.
45. Dhallan R, Au WC, Mattagajasingh S, et al. Methods to increase the percentage of free fetal DNA recovered from the maternal circulation. JAMA. 2004; 291(9): 1114-1119.

46. Chung GTY, Chiu RWK, Chan KCA, et al. Lack of dramatic enrichment of fetal DNA in maternal plasma by formaldehyde treatment. Clin Chem. 2005; 51(3): 655-658.

47. Chinnapapagari SKR, Holzgreve W, Lapaire O, et al. Treatment of maternal blood samples with formaldehyde does not alter the proportion of circulatory fetal nucleic acid (DNA and RNA) in maternal plasma. Clin Chem. 2005; 51(3): 652-655.

48. Li Y, Zimmermann B, Rusterholz C, et al. Size separation of circulatory DNA in maternal plasma permits ready detection of fetal DNA polymorphisms. Clin Chem. 2004; 50(6): 1002-1011.

49. Chan KCA, Zhang J, Hui ABY, et al. Size distributions of maternal and fetal DNA in maternal plasma. Clin Chem. 2004; 50(1): 88-92.

50. Li Y, Di Naro E, Vitucci A, et al. Detection of paternally inherited fetal point mutations for $\beta$-thalassemia using size-fractionated cell-free DNA in maternal plasma. JAMA. 2005; 293(7): 843-849.

51. Li J, Wang L, Mamon H, et al. Replacing PCR with COLD-PCR enriches variant DNA sequences and redefines the sensitivity of genetic testing. Nat Med. 2008; 14(5): 579-584.

52. Li J, Makrigiorgos GM. COLD-PCR: a new platform for highly improved mutation detection in cancer and genetic testing. Biochem Soc Transactions. 2009; 37(2): 427-432.

53. Kolialexi A, Tsangaris GT, Papantoniou N, et al. Application of proteomics for the identification of differentially expressed protein markers for Down syndrome in maternal plasma. Prenat Diagn. 2008; 28(8): 691-698.

54. Choolani M, Narasimkhan K, Kolla V, Hahn S. Proteomic technologies for prenatal diagnostics: advances and challenges ahead. Expert Rev Proteomics. 2009; 6: 87-101.

55. Tsangaris GT, Karamessinis P, Kolialexi A, et al. Proteomic analysis of amniotic fluid in pregnancies with Down syndrome. Proteomics. 2006; 6(15): 4410-4419.

56. Nagalla SR, Canick JA, Jacob T, et al. Proteomic analysis of maternal serum in Down syndrome: identification of novel protein biomarkers. J Proteome Res. 2007; 6: 1245-1257.

57. Kolla V, Jenö P, Moes S, et al. Quantitative proteomics analysis of maternal plasma in Down syndrome pregnancies using isobaric tagging reagent (iTRAQ). J Biomed Biotechnol. 2010; 2010: 952047 [Epub 2009 Nov 5]. 
58. Heywood WE, Madgett TE, Wang D, et al. 2D-DIGE analysis of maternal plasma for potential biomarkers for Down syndrome. Proteome Sci. 2011; 9: 56.

59. Heywood WE, Mills K, Wang D, et al. Identification of new biomarkers for Down's syndrome in maternal plasma. J Proteomics. 2012; in press.

60. Heywood WE, Wang D, Madgett TE, et al. The development of a peptide -SRM based tandem mass spectrometry assay for prenatal screening of Down syndrome. J Proteomics. 2012; in press. 